\title{
Impact of food-bound malabsorption on vitamin B12 status in older adults from the TUDA Ageing Cohort Study: preliminary findings
}

\author{
M. Clements $^{1}$, M. Ward ${ }^{1}$, C.F. Hughes ${ }^{1}$, L. Hoey ${ }^{1}$, A. Johnston ${ }^{1}$, A.M. Molloy ${ }^{2}$, \\ C. Cunningham $^{3}$, M.C. Casey ${ }^{3}$, F. Tracey ${ }^{4}$, M. O'Kane ${ }^{5}$, J.J. Strain ${ }^{1}$ and H. McNulty ${ }^{1}$ \\ ${ }^{1}$ Nutrition Innovation Centre for Food and Health (NICHE), Ulster University, Coleraine, United Kingdom, \\ ${ }^{2}$ School of Medicine, Trinity College Dublin, Dublin, Ireland, \\ ${ }^{3}$ Mercers Institute for Research on Ageing, St James's Hospital, Dublin, Ireland, \\ ${ }^{4}$ Causeway Hospital, Northern Health and Social Care Trust, Coleraine, United Kingdom and \\ ${ }^{5}$ Clinical Chemistry Laboratory, Western Health and Social Care Trust, Altnagelvin Hospital, Londonderry, \\ United Kingdom
}

\begin{abstract}
Vitamin B12 deficiency is common among older adults, even with dietary intakes well in excess of current recommendations. Severe clinical B12 deficiency (i.e. pernicious anaemia) leads to irreversible neurological damage, but once diagnosed, can be treated effectively with B12 injections. A much more common cause of low vitamin B12 status in older adults is food-bound malabsorption owing to atrophic gastritis. This in turn leads to reduced gastric acid secretion, thus limiting B12 absorption from food (given the essential role of gastric acid in releasing B12 from food proteins). Proton pump inhibitor (PPI) drugs reduce gastric acid secretion, similar to atrophic gastritis, thus there is a concern that these medications may lead to vitamin B12 malabsorption. Therefore, the aim of this study was to investigate biomarker status of vitamin B12 in relation to atrophic gastritis and PPI usage. Data were accessed from The Trinity Ulster Department of Agriculture (TUDA) Ageing Cohort Study, a cross-sectional study of community-dwelling adults ( $n$ $5186, \geq 60$ years) recruited across Northern Ireland and the Republic of Ireland (2008-2012). TUDA participants were classified into 3 groups; 'healthy' controls, atrophic gastritis and PPI users. Vitamin B12 status was assessed using a total of four biomarkers: serum total B12; serum holotranscobalamin, holoTC; plasma methylmalonic acid, MMA; plasma homocysteine. Atrophic gastritis was identified using pepsinogen analysis (via ELISA), with a pepsinogen I : II ratio of $<3$ considered indicative of atrophic gastritis. Based on results from all four biomarkers, participants with atrophic gastritis were found to have significantly lower B12 status compared to healthy controls: e.g. mean $(95 \% \mathrm{CI})$ serum total vitamin B12, $188(156,218) \mathrm{pmol} / \mathrm{L}$ vs. $262(252,272) \mathrm{pmol} / \mathrm{L} P<0.001$; holoTC, $46.0(38.1,53.8) \mathrm{pmol} / \mathrm{L}$ vs. $60.3(57.8,62.8) \mathrm{pmol} / \mathrm{L} P<0.001$; plasma MMA, $0.65(0.52,0.78) \mu \mathrm{mol} / \mathrm{L}$ vs. $0.37(0.32,0.42)$ $\mu \mathrm{mol} / \mathrm{L} P=0.001$. No differences in B12 biomarker concentrations were observed between PPI users and healthy controls. Regular consumption of fortified foods (i.e. $\geq 5$ portions per week) compared to non-regular consumption (i.e. $0-4$ portions per week) impacted positively on B12 biomarker status in all participants. This effect however appeared insufficient to restore normal vitamin B12 status in those with atrophic gastritis. These results show that older adults with atrophic gastritis have significantly lower vitamin B12 biomarker status, particularly in those who did not regularly consume fortified foods. Further investigations of the effect of atrophic gastritis and PPI usage on B12 status are warranted.
\end{abstract}

\section{Conflict of Interest}

There is no conflict of interest 